\title{
TIRBANIBULIN OINTMENT 1\% FOR ACTINIC KERATOSIS (AK): POOLED DATA FROM TWO PHASE 3 STUDIES
}

Andrew Blauvelt ${ }^{1}$, Steven Kempers ${ }^{2}$, Todd Schlesinger ${ }^{3}$, Edward Lain ${ }^{4}$, Hui Wang ${ }^{5}$, David Cutler ${ }^{5}$, Mark Lebwohl ${ }^{6}$, Jane Fang ${ }^{5}$, Rudolf Kwan ${ }^{5}$

\section{OPSIS}

- Tirbanibulin is a novel inhibitor of tubulin polymerization, also associated with disruption of Src kinase signaling, developed as a topical formulation for AK. W vehicle in AK clearance at 2 months post-treatment in two Phase 3 studies (FCD 2019).

\section{OBJECTIVE}

- Here we present pooled data analyses on efficacy, safety and 1-year follow-up.

\section{METHODS}

- Two identical Phase 3 randomized, double-blinded, vehicle-controlled studies evaluated efficacy and safety of tirbanibulin ointment $1 \%$ vs. vehicle in adults with AK on face/scalp.

- Eligible subjects with 4-8 clinically visible AK lesions in a $25 \mathrm{~cm}^{2}$ area were randomized $1: 1$ to receive tirbanibulin or vehicle (5-day once-daily seltapplication).

Primary and secondary endpoints were complete (100\%) and partial ( $\geq 75 \%)$ clearance of AK lesions at Day (D) 57.

- Safety including adverse events (AEs) and local skin reactions (LSRs; Grade O[none]-3[severe]) was assessed up to D57. Composite LSR scores represents the grades sum of all 6 LSR categories with a possible range from 0 to 18 .

- Subjects with complete AK clearance at D57 were followed for 1-year to assess safety and clearance durability.

\section{RESULTS}

Eligible subjects, predominantly Caucasian males with mean age of 70 , skin type III and had median of $6 \mathrm{AK}$ in the treatment area, were randomized to receive tirbanibulin (n=353) or velicle ointment $(n=349)$. Over $99 \%$ completed trealment. Baseline characteristics are shown in Table 1.

Table 1. Baseline characteristics

\begin{tabular}{lcc} 
& Tirbanibulin (n=353) & Vehicle $(\mathbf{n}=\mathbf{3 4 9})$ \\
\hline Mean Age (SD), years & $69.3(8.61)$ & $70.2(9.13)$ \\
Gender: Male, $\mathbf{n}(\%)$ & $305(86)$ & $304(87)$ \\
Race: White, $\boldsymbol{n}(\%)$ & $352(>99)$ & $348(>99)$ \\
Fitzpatrick Skin Type, $\mathbf{n}(\%)$ & & \\
Type I & $49(14)$ & $38(11)$ \\
Type II & $200(57)$ & $224(64)$ \\
Type III & $88(25)$ & $79(23)$ \\
Type IV & $15(4)$ & $7(2)$ \\
Type V & 0 & $1(<1)$ \\
Type VI & $1(<1)$ & 0 \\
Myedian Baseline AK lesion count (min - max) & $6.0(4-8)$ & $6.0(4-8)$ \\
\hline AK, actinic keratosisis SD, standard deviation & &
\end{tabular}
- At D57, complete clearance rates were significantly higher with tirbanibulin vs. respectively ( $\mathrm{P}<0.0001$ ) (Figure 1). Median reduction in AK lesion count at D57 was greater with tirbanibulin vs. vehicle $(87.5 \%$ vs. $20 \%$ )

Figure 1. Complete and partial clearance rates of AK lesions (ITT population) Complete clearance

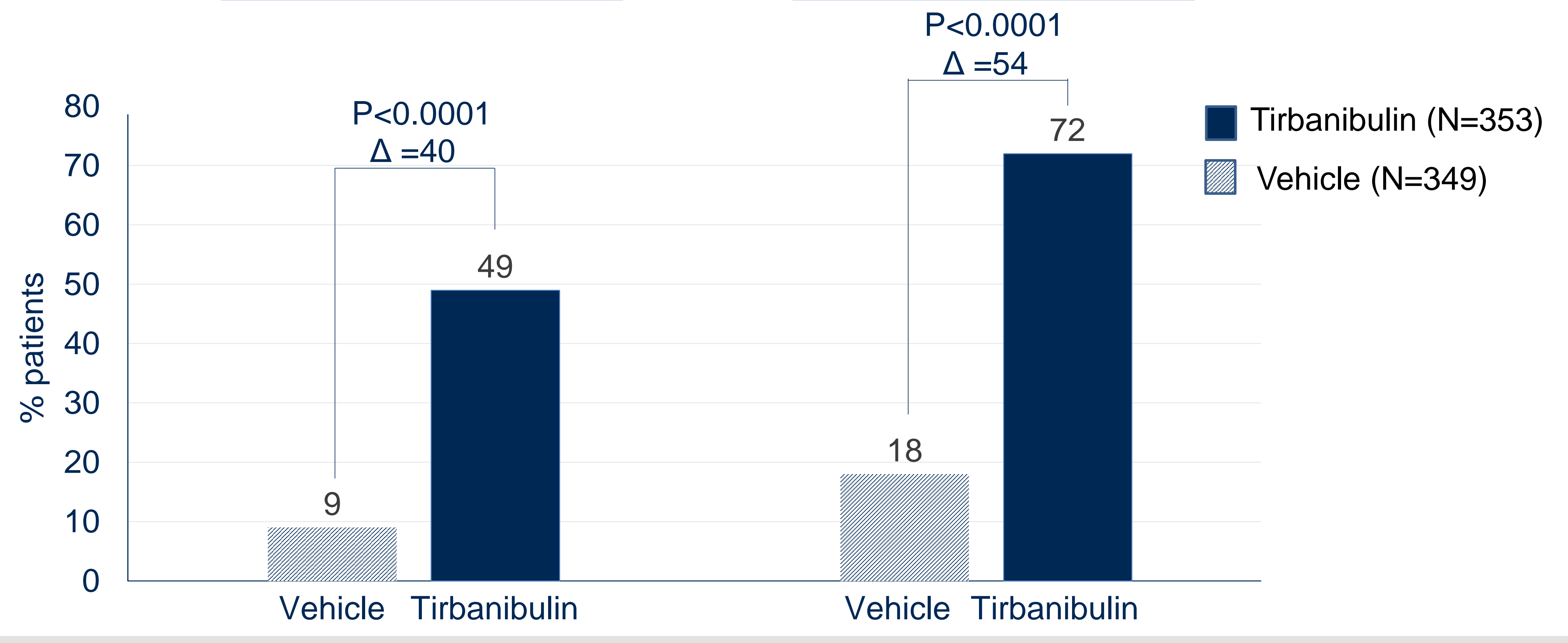

- AK lesion count to Day 57 is shown in Figure 2. Reduction in AK lesion count to (Table 2).

Figure 2. Number of lesions by visit and treatment group up to Day 57

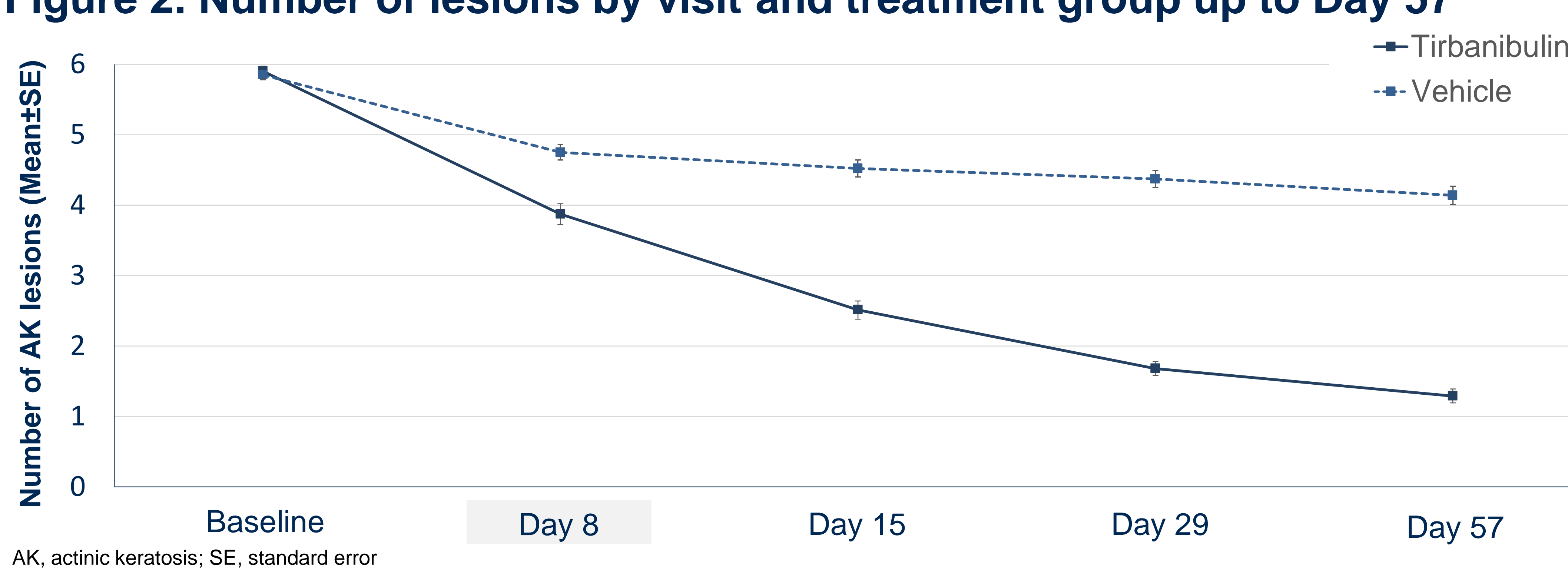

Baseline
Table 2. Summary of AK Lesion Counts Up to Day 57

\begin{tabular}{|c|c|c|c|}
\hline & & Tirbanibulin ( $\mathrm{n}=353)$ & Vehicle $(\mathbf{n}=349)$ \\
\hline Baseline & Mean (士SE) & $5.90(0.07)$ & $5.85(0.07)$ \\
\hline Day 8 & Mean (士SE) & $3.87(0.15)$ & $4.75(0.11)$ \\
\hline & Change from baseline, mean $(\%)$ & \multirow{2}{*}{\multicolumn{2}{|c|}{$<0.0001$}} \\
\hline & $p$-value & & \\
\hline Day 15 & Mean (土SE) & $2.51(0.13)$ & $4.52(0.12)$ \\
\hline & $\begin{array}{l}\text { Change from baseline } \\
\text { p-value }\end{array}$ & \multicolumn{2}{|c|}{$<0.0001$} \\
\hline Day 29 & Mean (士SE) & $1.68(0.10)$ & $4.37(0.12)$ \\
\hline & Change from baseline & \multicolumn{2}{|r|}{ - $1.48(-26 \%)$} \\
\hline Day 57 & Mean ( \pm SE) & $1.29(0.10)$ & $4.14(0.13)$ \\
\hline & Change from baseline & $-4.61(-79 \%)$ & $-1.72(-31 \%)$ \\
\hline
\end{tabular}

- At 1-year post-D57 follow-up, Kaplan-Meier estimate of proportion of tirbanibulintreated patients $(n=174)$ with at least one recurrent lesion present at baseline in the treated area recurring during follow-up was $47 \%$ and estimated rate of subjects with any AK lesion (recurred or new) was $73 \%$ (Figure 3). A total of $27 \%$ of patients had sustained AK clearance at 1-year.

Figure 3. Proportion of patients with any recurrence by number of lesions at baseline

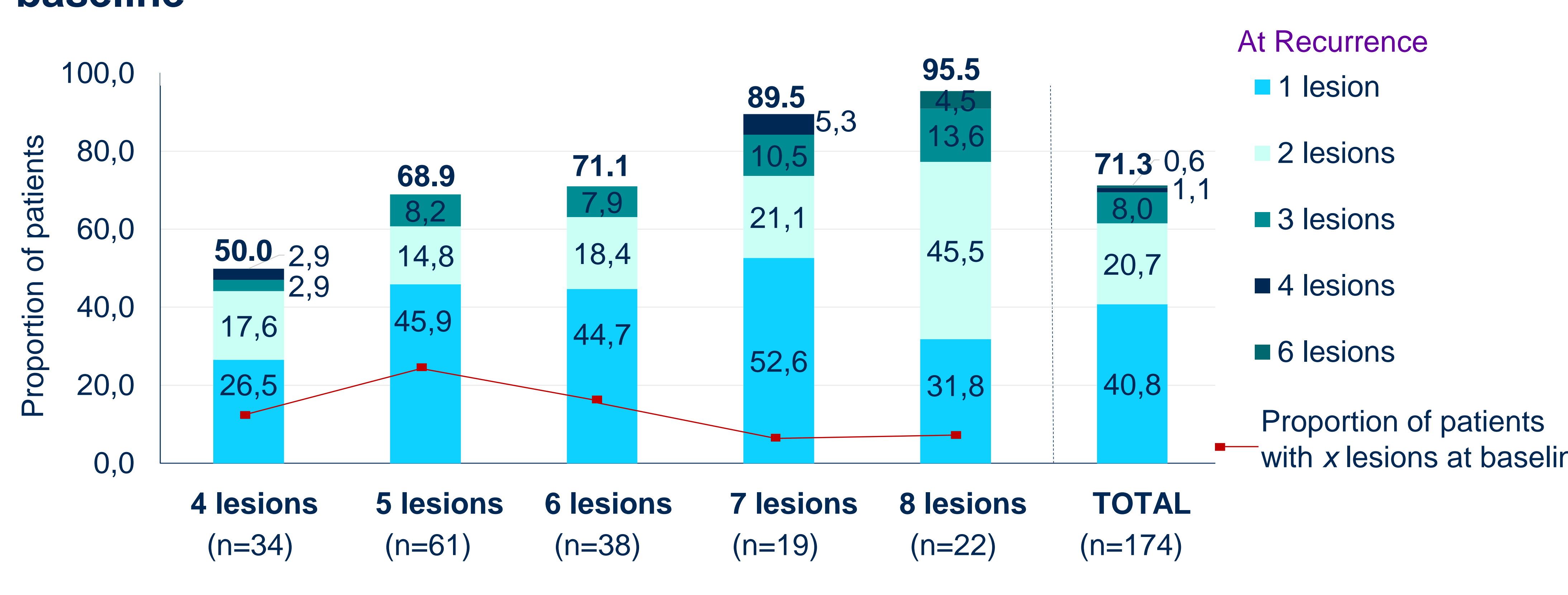

- Treatment-related AEs were few and mostly mild transient application-site pruritus (tirbanibulin vs. vehicle: $9 \%$ vs 6\%) and pain (tirbanibulin vs vehicle: $10 \%$ vs $3 \%$ ) (Table 3).

Table 3. Treatment-Related Adverse Events Up to Day 57 (Safety Population)

$n(\%)$ Safety population $(\mathrm{n}=702)$ \begin{tabular}{|l|l|l|}
\hline Application site pain & $35(10 \%)$ & $11(3 \%)$ \\
\hline
\end{tabular}

\begin{tabular}{|l|l|l|}
\hline Application site pruritus & $32(9 \%)$ & $21(6 \%)$ \\
\hline
\end{tabular}

- LSR signs were present at baseline, increased after treatment, peaked on D8 with tirbanibulin, decreased significantly by D15, and mostly resolved by D29.

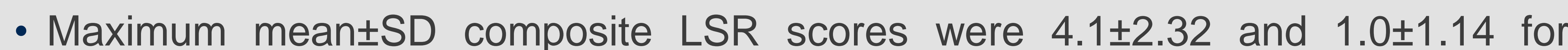
tirbanibulin and vehicle group, respectively.

- LSRs were mostly transient mild or moderate erythema and flaking/scaling. Severe LSRs were few. All LSRs resolved or returned to baseline and did not require intervention

No deaths, discontinuations, or serious AEs related to tirbanibulin occurred. - No treatment-related AEs throughout 1-year follow-up were reported.

\section{CONCLUSIONS}

- Tirbanibulin ointment $1 \%$ applied for 5 days was well tolerated, safe and effective potentially making it a valuable new addition to AK treatment.

\section{ACKNOWLEDGEMENTS}

